PROCEEDINGS OF THE

AMERICAN MATHEMATICAL SOCIETY

Volume 28, Number 2, May 1971

\title{
SEMIGROUPS ON ACYCLIC PLANE CONTINUA
}

\author{
B. E. WILDER
}

ABSTRACT. It is shown that an acyclic irreducible plane continuum which admits the structure of a topological semigroup is an arc if it has an identity, and is either an arc, is trivial, or is decomposible into an arc if it satisfies $M^{2}=M$. This extends some.results of Friedberg and Mahavier concerning semigroups on chainable continua.

Let $M$ be a topological semigroup with minimal ideal $K$ whose underlying space is a nondegenerate compact metric continuum. If $M$ has an identity, $M$ is called a clan.

Under the assumption that $M$ is chainable, Friedberg and Mahavier [3] showed that if $M$ is a clan it is an arc, and if $M^{2}=M$ then either $M$ is trivial, $M$ is an arc, or $M \mid K$ is an arc and $M$ is irreducible from a one-sided identity to some point. In this note we extend these results (using essentially the same arguments) by replacing the condition that $M$ be chainable by the condition that $M$ be an acyclic (i.e., contains no simple closed curve) plane continuum which is irreducible between two points. (Every nondegenerate chainable continuum is homeomorphic to such a continuum.)

THEOREM 1. If $M$ is an acyclic clan in the plane, then $M$ is arcwise connected.

Proof. Let $G$ be a closed subgroup of $M$ with identity $e$ and let $C(e)$ be the component of $G$ containing $e . C(e)$ is a subcontinuum of $M$ and is a group. Suppose $C(e)$ is nondegenerate. Then it is homogeneous and by [4] contains an arc; so by [1] it is a simple closed curve, contradicting the assumption that $M$ is acyclic. Thus $C(e)$ is degenerate and $G$ is totally disconnected. Then $M$ is arcwise connected by [6].

COROLlaRy. If $M$ is an acyclic plane continuum which is irreducible between two of its points it is an arc.

REMARK. The referee has observed that except for the existence of the one-sided identity, the conclusion of the next theorem follows from Hunter's argument in [5, Theorem 8], without the assumption

Received by the editors May 3, 1970.

AMS 1969 subject classifications. Primary 5455; Secondary 5480.

Key words and phrases. Topological semigroup, clan, acyclic plane continua, arcwise connected. 
that $M$ be acyclic. Also, a simplification suggested by the referee has been employed in the next argument.

THEOREM 2. If $M$ is an acyclic plane continuum which is irreducible between two of its points and $M^{2}=M$, then either

(1) $M=K$ and the multiplication on $K$ is trivial,

(2) $M$ is an arc, or

(3) $M$ has a one-sided identity e, $M \mid K$ is an arc, and $M$ is irreducible from e to some point.

Proof. Let $E$ denote the set of idempotent elements of $M$, and for $e$ in $E$, let $H_{e}$ be the maximal subgroup containing $e$. Since $M$ is acyclic, $K$ is not the cartesian product of two nondegenerate continua [5, Lemma 2, p. 238]; so $K$ is a group or multiplication in $K$ is trivial [7, Corollary 1]. As in the proof of Theorem 1, if $K$ is a group it is degenerate. In either case multiplication in $K$ is trivial and $K$ is a subset of $E$.

Now assume that $M \neq K$ and $M$ is not an arc. Suppose $M$ has no one-sided identity. Since $M$ is irreducible between two points $a$ and $b$, there exist points $e$ and $f$ in $E \backslash K$ such that $a \in H_{e}, b \in H_{f}, H_{e}$ and $H_{f}$ are connected, and $M=(e M e) \cup(f M f)$ [7, Theorem 5]. But $H_{e}$ and $H_{f}$ are degenerate so $M$ is irreducible from $e$ to $f$. Since $e M e$ and $f M f$ are acyclic plane clans, they are arcwise connected by Theorem 1. Then $M$ is an arc from $e$ to $f$, a contradiction. Thus $M$ has a right (or left) identity $e$.

Then $M e=M$ and $e M=e M e$ is either degenerate or arcwise connected. If $e M$ is degenerate, $e \in K$ and $M e=M=K$, a contradiction. Hence $e M=e M e$ is a nondegenerate arcwise connected clan with $e$ as its identity. Let $T$ be an arc in $e M$ from $e$ to its minimal ideal $K^{\prime}$ such that $T \cap K^{\prime}$ is degenerate. Clearly $K^{\prime} \subseteq K$. Since each of $a T$ and $b T$ is a continuous image of $T$, each is either degenerate or arcwise connected, and there is an $\alpha$ and a $\beta$ such that each of $\alpha$ and $\beta$ is an arc or degenerate, $\alpha \subseteq a T, \beta \subseteq b T, \alpha$ contains $a, \beta$ contains $b$ and each of $\alpha$ and $\beta$ intersects $K$ at only one point. Since $M$ is irreducible from $a$ to $b, M=\alpha \cup K \cup \beta$. If both $a$ and $b$ belong to $K, K=M$, so let $e \in \beta \backslash K$. If $e \neq b, e$ possesses a euclidean (1-dimensional) neighborhood and since $e$ is a right identity, $e \in K$, a contradiction [2, Lemma $4]$. Hence $e=b$ and (3) holds.

REMARK. An application of Theorem 1 to some nonchainable continua would be as follows: no continuum in the plane consisting of an infinite half-ray "spiraling down" upon a nondegenerate acyclic continuum admits the structure of a topological semigroup with identity. 


\section{REFERENCES}

1. R. H. Bing, A simple closed curve is the only homogeneous bounded plane continuum that contains an arc, Canad. J. Math. 12 (1960), 209-230. MR 22 \#1869.

2. Haskell Cohen and R. J. Koch, Acyclic semigroups and multiplications on two-manifolds, Trans. Amer. Math. Soc. 118 (1965), 420-427. MR 30 \#5283.

3. M. Friedberg and W. S. Mahavier, Semigroups on chainable and circle-like continua, Math. Z. 106 (1968), 159-161. MR 38 \#263.

4. A. M. Gleason, Arcs in locally compact groups, Proc. Nat. Acad. Sci. U.S.A. 36 (1950), 663-667. MR 12, 391.

5. R. P. Hunter, Note on arcs in semigroups, Fund. Math. 49 (1960/61), 233-245. MR 23 \#A628.

6. R. J. Koch, Threads in compact semigroups, Math. Z. 86 (1964), 312-316. MR 30 \#1499.

7. R. J. Koch and A. D. Wallace, Admissibility of semigroup structures on continua, Trans. Amer. Math. Soc. 88 (1958), 227-287. MR 20 \#1729.

Tennessee Technological University, Cookeville, Tennessee 38501 